\title{
Dyslipidaemia Associated with the Highly Active Antiretroviral Therapy in AIDS Patient: Reversion After Switching (Stavudine to Tenofovir and Lopinavir/Ritonavir to Atazanavir/Ritonavir)
}

\author{
Hamilton Domingos, Rivaldo Venâncio da Cunha and Anamaria Mello Miranda Paniago \\ Federal University of Mato Grosso do Sul; Campo Grande, MS, Brazil
}

\begin{abstract}
Antiretroviral therapy has been associated with hyperlipidemia in AIDS patients. This case illustrates the classic metabolic effects associated to the HAART including protease inhibitors and/or stavudine. It was showed that the management of the HAART-associated dyslipidaemia with conventional antihyperlipidemic therapy may fail, being the switching strategy the best option.
\end{abstract}

Key Words: AIDS, dyslipidaemia, switching strategy.

Highly active antiretroviral therapy (HAART), in combinations including protease inhibitors (PIs), has achieved sustained suppression of HIV replication and reduced morbidity and mortality rates in patients with advanced HIV infection [1].

Dyslipidaemia, which includes hypercholesterolemia, hypertriglyceridemia and mixed lipid disorders, is a disturbance frequently observed in persons with HIV infection. Many antiretroviral drugs induce changes in lipid profiles. Protease inhibitors (PIs) seem most consistently to affect lipid levels compared with other antiretroviral classes. However, elevation of cholesterol has been observed with the use of efavirenz and nucleoside reverse transcriptase inhibitors (nRTIs), such as stavudine [2].

Typically, the lipid profile of patients infected with HIV, who were receiving protease inhibitors, is characterized by the combined effects of the patient's underlying disease (HIV) and antiretroviral pharmacotherapy (protease inhibitors). Serum levels of total cholesterol, triglycerides and LDLcholesterol are usually elevated, while HDL-cholesterol levels are often reduced $[3,4]$.

Fat redistribution or lipodystrophy, hypertriglyceridemia, hypercholesterolemia, insulin resistance and diabetes mellitus have been extensively reported in subjects treated with protease inhibitor-based antiretroviral regimens. In particular, dyslipidaemia occurs in up to $70-80 \%$ of HIV-infected individuals receiving HAART and can be associated with all the available PIs, although hypertriglyceridemia seems to be more frequent in patients treated with ritonavir, ritonavir/ saquinavir, or ritonavir/lopinavir. The potential long term consequences of HAART-associated hyperlipidemia are not completely understood, but an increased risk of premature coronary artery disease has been reported in young HIVpositive persons receiving PIs [5].

Received on 24 October 2006; revised 4 March 2007.

Address for correspondence: Dr. Hamilton Domingos, R.Quintino Bocaiuva, 1014. TV Morena, Zip code: 79050-112, Campo Grande, Mato Grosso do Sul, Brazil. Phone: 0xx67-3342-5563, FAX: 0xx 673383-3053. E-mail - hmdomingos@brturbo.com.br.

The Brazilian Journal of Infectious Diseases

2007;11(2):290-292. (C) 2007 by The Brazilian Journal of Infectious Diseases and Contexto Publishing. All rights reserved.
With regard to newer PIs, the real incidence of metabolic abnormalities has not been defined yet for amprenavir, whereas the occurrence of hyperlipidemia seems more frequent in subjects receiving a lopinavir/ritonavir-based treatment [6-8]. On the other hand, preliminary studies have shown that atazanavir, a novel azapeptide PI, is not associated with increase in total cholesterol, LDL cholesterol, or triglyceride serum levels [9].

\section{Case Report}

A 34 year-old man, diagnosed with AIDS on 25/08/1997. The HAART was initiated including zidovudine, lamivudine and didanosine, being maintained in normal limits the metabolic parameters: total cholesterol $=130.0 \mathrm{mg} / \mathrm{dL}$, HDLcholesterol $=40.8 \mathrm{mg} / \mathrm{dL}$, LDL-cholesterol $=65.4 \mathrm{mg} / \mathrm{dL}$, triglycerides $=119.0 \mathrm{mg} / \mathrm{dL}$ and glucose $=93 \mathrm{mg} / \mathrm{dL}$ and the patient had $\mathrm{CD}_{4}$ lymphocytes $=103$ cells $/ \mathrm{mm}^{3}$ and viral load $=400$ copies $/ \mathrm{mm}^{3}$.

The HAART was modified to stavudine, lamivudine and efavirenz on 31/08/2000, due to therapeutic failure, expressed by $\mathrm{CD}_{4}$ lymphocytes $=109$ cells $/ \mathrm{mm}^{3}$ and viral load $=7,300$ copies $/ \mathrm{mm}^{3}$. There was increase of the total cholesterol $=209.0$ $\mathrm{mg} / \mathrm{dL}$, of the LDL- cholesterol=115.0 $\mathrm{mg} / \mathrm{dL}$, of the triglycerides $=274.0 \mathrm{mg} / \mathrm{dL}$, and of glucose $=100 \mathrm{mg} / \mathrm{dL}$.

On 16/07/2002, with $\mathrm{CD}_{4}$ lymphocytes $=64$ cells $/ \mathrm{mm}^{3}$ and viral load $=68,000$ copies $/ \mathrm{mm}^{3}$, there was new modification in HAART, being switched efavirenz to lopinavir/ritonavir. The patient evolved with immunologic and virologic improvement $\left(C_{4}\right.$ lymphocytes $=193 \mathrm{cell} / \mathrm{mm}^{3}$ and viral load $=80$ copies $\left./ \mathrm{mm}^{3}\right)$, however, there was significative worsening of metabolic parameters: total cholesterol $=346.0 \mathrm{mg} / \mathrm{dL}$, HDLcholesterol $=26.0 \mathrm{mg} / \mathrm{dL}$, triglycerides $=2,462.0 \mathrm{mg} / \mathrm{dL}$ and glucose $=124 \mathrm{mg} / \mathrm{dL}$.

It was prescribed antihyperlipidemic therapy (bezafibrate $400 \mathrm{mg}$ /day) being observed the following results: total cholesterol=263.0 mg/dL, HDL-cholesterol=22.0 mg/dL, triglycerides $=1,314.0 \mathrm{mg} / \mathrm{dL}$ and glucose $=99 \mathrm{mg} / \mathrm{dL}$.

Subsequent laboratory investigations, without bezafibrate, showed triglycerides and cholesterol levels of $4,152.0 \mathrm{mg} / \mathrm{dL}$ and $418.0 \mathrm{mg} / \mathrm{dL}$, respectively. The abdominal ultrasonography showed hepatic steatosis.

Antihyperlipidemic therapy was initiated again, but with another fibrate, ciprofibrate $200 \mathrm{mg}$ /day associated with 
Table 1. Metabolic and immunological parameters with different HAART regimens and different therapeutics approaches

\begin{tabular}{lcccccccc}
\hline HAART $/$ Parameters & $\mathbf{1}$ a. & $\mathbf{2}$ a. & $\mathbf{3}$ a. & $\mathbf{4}$ a. & $\mathbf{5}$ a. & $\mathbf{6}$ a. & $\mathbf{7}$ a. & $\mathbf{8}$ a. \\
\hline Total chol. (mg/dL) & 130 & 209 & 346 & 263 & 418 & 270 & 126 & 193 \\
HDL- chol. (mg/dL) & 41 & 39 & 26 & 22 & - & - & 34 & 49 \\
LDL- chol. (mg/dL) & 65 & 115 & - & - & - & - & 79 & 96 \\
Triglyc. (mg/dL) & 119 & 274 & 2462 & 1314 & 4152 & 478 & 68 & 242 \\
Glucose (mg/dL) & 93 & 100 & 124 & 99 & 118 & 101 & 102 & 125 \\
CD4 Lymph.(cell/mm3) & 103 & 109 & 193 & - & 268 & - & 213 & - \\
Viral Load (copies/mm3) & 400 & 7300 & 80 & - & 750 & - & 290 & - \\
\hline
\end{tabular}

HAART regimens and different phases of treatment:

1 a. phase: Zidovudine, lamivudine and didanosine. 2 a. phase: Stavudine, lamivudine and efavirenz. 3 a. phase: Stavudine, lamivudine and lopinavir/ritonavir. 4 a. phase: Stavudine, lamivudine, lopinavir/ritonavir and bezafibrate. 5 a. phase: Stavudine, lamivudine and lopinavir/ritonavir. 6 a. phase: Stavudine, lamivudine, lopinavir/ritonavir, ciprofibrate and nicotinic acid. 7 a. phase: Tenofovir, lamivudine, atazanavir/ritonavir and ciprofibrate. 8 a. phase: Tenofovir, lamivudine, atazanavir/ritonavir.

nicotinic acid $1,000 \mathrm{mg} /$ day. The patient was followed for 3 months, however, the improvement of the metabolic abnormalities was partial: total cholesterol=270.0 $\mathrm{mg} / \mathrm{dL}$, triglycerides $=478.0 \mathrm{mg} / \mathrm{dL}$ and glucose $=101 \mathrm{mg} / \mathrm{dL}$. In this phase of the HAART, CD lymphocytes were 268 cells $/ \mathrm{mm}^{3}$ and viral load was 750 copies/ $\mathrm{mm}^{3}$.

Due to the difficulty to control the lipid levels, the HAART was modified again, switching from stavudine to tenofovir and lopinavir/ritonavir to atazanavir/ritonavir, on September 13, 2005. After 6 months, still using ciprofibrate $100 \mathrm{mg} /$ day, the metabolic parameters evolved with significative improvement, being observed total cholesterol $=126.0 \mathrm{mg} / \mathrm{dL}$, HDL-cholesterol=34.0 mg/dL, LDL-cholesterol=79.0 mg/dL, triglycerides $=68.0 \mathrm{mg} / \mathrm{dL}$ and glucose $=102.0 \mathrm{mg} / \mathrm{dL}$.

After 2 months, the patient was evaluated without ciprofibrate, and the metabolic parameters observed were: total cholesterol=193.0 mg/dL, HDL-cholesterol=49.0 mg/dL, LDLcholesterol $=95.6 \mathrm{mg} / \mathrm{dL}$, triglycerides $=242.0 \mathrm{mg} / \mathrm{dL}$ and glucose $=125 \mathrm{mg} / \mathrm{dL}$.

\section{Discussion}

The use of protease inhibitors (PIs) has been associated with hyperlipidemia, which is more common and more severe than the observed before the advent of the HAART [10].

More recently, higher triglycerides levels were reported in subjects taking stavudine, compared with tenofovir-based regimen. The evaluation and management of dyslipidaemia is based on the future risk of developing syntomatic coronary artery disease [2].

This case illustrates the classic metabolic effects associated to the HAART, including protease inhibitors and/ or stavudine.

After lifestyle modifications and antihyperlipidemic therapy, no satisfactory results were obtained, without reaching total control of dyslipidaemia.

Antihyperlipidemic therapy frequently fails to return serum levels of total cholesterol and triglycerides to the normal limits. As such, coronary risks are likely to persist in a number of individuals with protease inhibitorsassociated hyperlipidemia. Therefore, discontinuing protease inhibitor therapy and replacing the offending agent with another less lipogenic medication may be beneficial in a number of patients [11].

It was demonstrated that the management of the dyslipidaemia associated to the HAART with conventional antihyperlipidemic therapy, pharmacologic and non pharmacologic, may fail, being the switching strategy the best option to these cases. It has the potential advantage of avoiding pharmacologic intervention for elevations in lipid levels [12].

Calza et al. found that the pharmacological treatment of the dyslipidaemia with pravastatina or bezafibrato associated to the permanence of the HAART had an antihyperlipidemic efficacy greater than the switching from the PI to nevirapina or efavirenz [13].

Switching from a PI to nevirapina or abacavir has generally resulted in an improvement in total cholesterol and triglycerides levels, whereas switching to efavirenz has produced less consistent results [14]. Studies of switches from stavudine to abacavir have yielded inconclusive results. These trials have generally demonstrated persistent viral suppression for 6-12 months after switching regimens [15-17].

Studies comparing the effects of treatment switching and the effects of adding lipid-lowering agents to ongoing successful therapy have not been reported. Clinicians will need to weigh the risks of new treatment-related toxicities and the possibility of virologic relapse when switching antiretroviral drugs. The risks of potential drug interactions and new treatment-related toxicities from lipid-lowering agents that are added to existing regimens must be evaluated too [10].

This case also demonstrates that many trials are necessary to show the best way to approach the patients with AIDS receiving HAART and presenting dyslipidaemia.

\section{References}

1. Green M.L. Evaluation and Management of Dyslipidaemia in patients with HIV infection. JGIM 2002;17:797-802.

2. Fichtenbaun C.J. Selected metabolic complications of HIV infection and their treatment - overview. International AIDS Society-USA 2006. 
3. Sellmeyer D.E., Grunfeld C. Endocrine and metabolic disturbances in human immunodeficiency virus infection and the acquired immune deficiency syndrome. Endocr Rev 1996;17(5):51832.

4. Penzak S.R., Chuck S.K. Hyperlipidemia associated with HIV protease inhibitor use: patophysiology, prevalence, risk factors and treatment. Scand J Infect Dis 2000;32:11-23.

5. Calza L., Manfredi R., Chiodo F. Dyslipidaemia associated with antiretroviral therapy in HIV-infected patients. Journal of Antimicrobial Chemotherapy 2004;53:10-14.

6. Fung H.B., Kirschenbaun H.L., Hameed R. Amprenavir: A new human immunodeficiency virus type I protease inhibitor. Clinical Therapeutics 2000; 22: 549-72.

7. Benson C.A., Deeks S.G., Brun S.C., et al. Safety and antiviral activity at 48 weeks of lopinavir/ritonavir plus nevirapina and two nucleoside reverse-transcriptase inhibitors in human immunodeficiency virus type I- infected protease inhibitor experienced patients. Journal of Infectious Diseases 2002; 185:599-607.

8. Gutierrez F., Padilla S., Navarro A., et al. Lopinavir plasma concentrations and changes in lipid levels during salvage therapy with lopinavir/ritonavir containing regimens. Journal of Acquired Immune Deficiency Syndromes 2003;33: 594- 600.

9. Goldsmith D.R., Perry C.M. Atazanavir. Drugs 2003;63:1679- 93

10. Dubé M.P., Stein J.H., Aberg J.A., et al. Guidelines for the evaluation and management of dyslipidaemia in human immunodeficiency virus (HIV)-infected adults receiving antiretroviral therapy: Recommendations of the HIV Medicine Association of the Infectious Disease Society of America and the Adult AIDS Clinical Trials Group. Clinical Infectious Diseases 2003;37:613-27.
11. Penzak S.R., Chuck S.K. Management of protease inhibitorassociated hyperlipidemia. Am J Cardiovasc Drugs 2002;2(2):91106.

12. Saag M.S., Powderly W.G., Schamelan M., et al. Switching antiretroviral drugs for treatment of metabolic complications in HIV-1 infection: summary of selected trials. Topics in HIV Medicine 2002;10:47-51.

13. Calza L., Manfredi R., Colangeli V., et al. Switching of a protease inhibitor to nevirapina or efavirenz compared to treatment to reduce lipids in the control of dyslipidaemia. AIDS 2005;19:1051-8.

14. Martinez E., Garcia-Viejo M.A., Blanco J.L., et al. Impact of switching from human immunodeficiency vírus type 1 protease inhibitors to efavirenz in successfully treated adults with lipodystrophy. Clin Infect Dis 2000;31:1266-73.

15. Carr A., Workman C., Smith D.E., et al. Abacavir Substitution for nucleoside analogs in patients with HIV lipoatrophy: A randomized trial. JAMA 2002;288:207-15.

16. John M., James I., Mckinnon E., et al. A randomized, controlled, open label study of revision of antiretroviral regimens containing stavudine and or a protease inhibitor to zidovudine/lamivudine/ abacavir to prevent or reverse lipoatrophy (abstract 700-T). In: Program and abstracts of the $9^{\text {th }}$ Conference on Retroviruses and Opportunistic Infections (Seattle). Alexandria, VA: Foundation for Retrovirology and Human Health, 2002: 308.

17. McComsey G., Lonergan T., Fisher R., et al. Improvements in lipodystrophy are observed after 24 weeks when stavudine is replaced by either abacavir or zidovudine (abstract 701-T). In: Program and abstracts of the $9^{\text {th }}$ Conference on Retroviruses and Opportunistic Infections (Seattle). Alexandria, VA: Foundation for Retrovirology an Human Health, 2002:309. 